\title{
CLASS-D AUDIO AMPLIFIERS WITH NEGATIVE FEEDBACK*
}

\author{
STEPHEN M. COX ${ }^{\dagger}$ AND BRUCE H. CANDY
}

\begin{abstract}
There are many different designs for audio amplifiers. Class-D, or switching, amplifiers generate their output signal in the form of a high-frequency square wave of variable duty cycle (ratio of on time to off time). The square-wave nature of the output allows a particularly efficient output stage, with minimal losses. The output is ultimately filtered to remove components of the spectrum above the audio range. Mathematical models are derived here for a variety of related class-D amplifier designs that use negative feedback. These models use an asymptotic expansion in powers of a small parameter related to the ratio of typical audio frequencies to the switching frequency to develop a power series for the output component in the audio spectrum. These models confirm that there is a form of distortion intrinsic to such amplifier designs. The models also explain why two approaches used commercially succeed in largely eliminating this distortion; a new means of overcoming the intrinsic distortion is revealed by the analysis.
\end{abstract}

Key words. class-D amplifier, total harmonic distortion, mathematical model

AMS subject classifications. 34E13, 37N20

1. Introduction. Class-D audio amplifiers are becoming increasingly popular, particularly at the high end of the hi-fi audio amplification market. The key feature of their design is that they switch their output between two voltage levels at a very high frequency (typically $500 \mathrm{kHz}$ ), well above the audio range. The audio signal is essentially encoded in the relative durations of the pulses at the two output voltage levels. The discrete nature of the switching then allows the output stage to be highly efficient; the audio signal is recovered by low-pass filtering of the output. Although the concept of class-D amplifiers using this pulse-width modulation (PWM) technique has been known for at least fifty years [1], it is only much more recently that electronic components have become available that make their practical implementation feasible. Several commercial amplifiers at the high end of the audio market use class-D amplifier technology.

In its simplest manifestation, the class-D amplifier is known to be capable of producing no distortion to audio signals $[1,4,5]$, at least when the mathematical model assumes, as we shall do, that electronic components perform in an ideal fashion, and that the circuit is free from noise. (Significant effort has also been applied to devising remedies for the effects of imperfections in the circuit components [2], for example, nonlinearities in a carrier waveform that is generally modelled mathematically as a piecewise-linear (triangular or sawtooth) wave [6].)

Unfortunately, the simplest design is prone to noise (including thermal and outputstage power-supply noise), due to a lack of negative feedback, and so more sophisticated versions of the class-D design have been developed, incorporating such feedback, in an attempt to counter the poor noise performance. While these negative-feedback designs do indeed have better noise performance, they also significantly distort the output, even with perfect components, and there have been various attempts to develop further the negative-feedback designs to counter this intrinsic distortion.

*This work was supported by Extraordinary Technology Pty Ltd, Australia. This work appeared in preliminary form in the proceedings of the 117th Audio Engineering Society Convention, San Francisco, October 2004.

$\dagger$ Corresponding author: School of Mathematical Sciences, University of Adelaide, Adelaide 5005, Australia (stephen.cox@adelaide.edu.au).

${ }^{\ddagger}$ Halcro, 118 Hayward Avenue, Torrensville 5031, Australia (brucec@adam.com.au). 
Despite the great practical value of the application, and the variety of 'engineering' solutions available, there appears to be a dearth of mathematical models for class$\mathrm{D}$ amplifier designs with negative feedback. By contrast, the no-feedback case was analysed over fifty years ago by Black in his treatise [1], and was shown there to allow distortion-free output of sinusoidal input signals. More recently the same problem was reconsidered in greater depth $[4,5]$ and it was shown that there is no distortion to any audio signal, sinusoidal or otherwise. The latter result is significant because the amplifier design is nonlinear, so the distortion characteristics of an arbitrary signal cannot be inferred from those of its Fourier components.

We develop mathematical models for class-D amplifiers with negative feedback. The models proceed from the governing differential equations that relate the voltage signals at the various parts of the device, assuming perfect components. The resulting system of equations may be formally integrated to yield what is essentially a set of nonlinear difference equations for the various internal signals at multiples of the switching period. The solution to these equations is then developed in an asymptotic series based on the separation of scales between the (relatively high-frequency) switching stage and the (relatively low-frequency) audio signal. The analysis is continued as far as the first term in the series that reveals the inherent distortion of the system. We then show how two successful commercial approaches to reducing significantly this component of the distortion can be modelled, and confirm what is already known empirically, that they do indeed work. The analysis reveals a third means of reducing the intrinsic distortion. We conclude by considering briefly the effects of nonlinear distortion to the carrier wave upon the audio output.

2. Mathematical model: general considerations. The 'classical' class-D amplifier design, without negative feedback, is illustrated in Figure 2.1. The audio input signal is denoted by $s(t)$; generally this signal comprises a Fourier spectrum in the audible range up to $20 \mathrm{kHz}$. This audio signal is added to a triangular carrier wave $v(t)$, with period $T$, that satisfies

$$
v(t)=\left\{\begin{array}{cc}
1-\frac{4 t}{T} & \text { for } 0 \leq t<\frac{T}{2} \\
-3+\frac{4 t}{T} & \text { for } \frac{T}{2} \leq t<T
\end{array}\right.
$$

and $v(t+T)=v(t)$ for all $t$. Thus $v(n T)=1$ and $v\left(\left(n+\frac{1}{2}\right) T\right)=-1$, for any integer $n$, and $v(t)$ is piecewise linear between these two values. It will be significant for the analysis that follows that if $\omega$ is a typical audio frequency then $\omega T \ll 1$. The main circuit element is a comparator, which compares the voltage at its noninverting input (denoted by a ' + ' in the figure) with the voltage at its inverting input (denoted by a '-'), and gives an output $g(t)$ that satisfies

$$
g(t)= \begin{cases}+1 & \text { if } s(t)+v(t)>0 \\ -1 & \text { if } s(t)+v(t)<0\end{cases}
$$

Note that the output voltages have been normalized to \pm 1 : furthermore, we assume throughout this paper that $-1<s(t)<1$ for all $t$. The switching times of $g(t)$ are thus governed by $s(t)+v(t)=0$; we denote the switching times from +1 to -1 by $t=n T+\alpha_{n}$, with the reverse switchings at times $t=n T+\beta_{n}$. For the 'classical' design in Figure 2.1 these switching times are governed by

$$
0<\alpha_{n}=\frac{T}{4}\left(1+s\left(n T+\alpha_{n}\right)\right)<\frac{T}{2}<\beta_{n}=\frac{T}{4}\left(3-s\left(n T+\beta_{n}\right)\right)<T .
$$




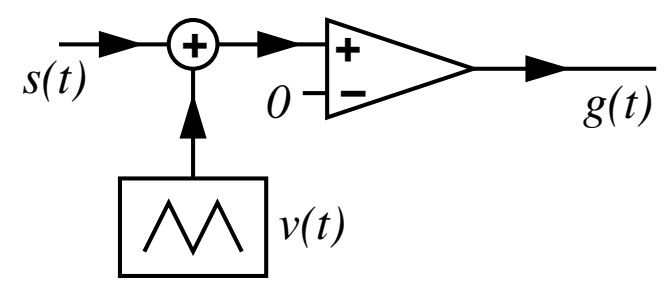

FiG. 2.1. 'Classical' class-D amplifier (without negative feedback). The audio input signal is $s(t)$; this is summed with a high-frequency triangular carrier wave $v(t)$ and input to the noninverting input (+) of a comparator, whose inverting input (-) is grounded. The output of the comparator is $g(t)$, given by (2.2).

Note that the equations in (2.3) give $\alpha_{n}$ and $\beta_{n}$ only implicitly. We shall consider their solution later.

We now examine how the switching times are used in computing the component of $g(t)$ in the audio spectrum, i.e., the amplifier output.

2.1. Comparator output $g(t)$. In all class-D designs, regardless of the details, the output $g(t)$ takes the form

$$
g(t)= \begin{cases}+1 & \text { if } n T<t<n T+\alpha_{n} \text { or } n T+\beta_{n}<t<(n+1) T \\ -1 & \text { if } n T+\alpha_{n}<t<n T+\beta_{n}\end{cases}
$$

for some switching times $t=n T+\alpha_{n}$ and $n T+\beta_{n}$. Thus we may write

$$
g(t)=1-2 \sum_{n=-\infty}^{\infty} \mathcal{H}_{n}(t)
$$

where

$$
\mathcal{H}_{n}(t)=H\left(t-\left(n T+\alpha_{n}\right)\right)-H\left(t-\left(n T+\beta_{n}\right)\right),
$$

and where $H(t)$ is the Heaviside step function $(H(t)=0$ for $t<0$ and $H(t)=1$ for $t>0)$. Note that each $\mathcal{H}_{n}(t)$ has finite support:

$$
\mathcal{H}_{n}(t)= \begin{cases}1 & n T+\alpha_{n}<t<n T+\beta_{n} \\ 0 & \text { otherwise }\end{cases}
$$

so the sum in (2.5) is well defined almost everywhere (i.e., except at the exact switching times). The corresponding Fourier transform

$$
\hat{g}(\omega) \equiv \frac{1}{(2 \pi)^{1 / 2}} \int_{-\infty}^{\infty} g(t) \mathrm{e}^{-\mathrm{i} \omega t} \mathrm{~d} t
$$

is then

$$
\hat{g}(\omega)=(2 \pi)^{1 / 2} \delta(\omega)-\frac{2 \mathrm{i}}{(2 \pi)^{1 / 2} \omega} \sum_{n=-\infty}^{\infty}\left\{\mathrm{e}^{-\mathrm{i} \omega\left(n T+\beta_{n}\right)}-\mathrm{e}^{-\mathrm{i} \omega\left(n T+\alpha_{n}\right)}\right\} .
$$

A formal analysis of this expression allows us to write the output $g(t)$ more usefully in terms of the switching times. We begin by denoting by $\mathcal{S}$ the sum in (2.9). Then

$$
\mathcal{S}=\sum_{n=-\infty}^{\infty} \mathrm{e}^{-\mathrm{i} \omega n T}\left(\mathrm{e}^{-\mathrm{i} \omega \beta_{n}}-\mathrm{e}^{-\mathrm{i} \omega \alpha_{n}}\right)
$$




$$
\begin{aligned}
& =\sum_{n=-\infty}^{\infty} \mathrm{e}^{-\mathrm{i} \omega n T} \sum_{m=1}^{\infty} \frac{1}{m !}(-\mathrm{i} \omega)^{m}\left(\beta_{n}^{m}-\alpha_{n}^{m}\right) \\
& =\sum_{m=1}^{\infty} \frac{1}{m !}(-\mathrm{i} \omega)^{m} \sum_{n=-\infty}^{\infty} \mathrm{e}^{-\mathrm{i} \omega n T}\left(\beta_{n}^{m}-\alpha_{n}^{m}\right) .
\end{aligned}
$$

Now we note that

$$
\mathrm{e}^{-\mathrm{i} \omega n T}\left(\beta_{n}^{m}-\alpha_{n}^{m}\right)=\int_{-\infty}^{\infty} \mathrm{e}^{-\mathrm{i} \omega t}\left[B^{m}(t)-A^{m}(t)\right] \delta(t-n T) \mathrm{d} t,
$$

where $A(t)$ and $B(t)$ are any smooth functions that satisfy

$$
A(n T)=\alpha_{n}, \quad B(n T)=\beta_{n} .
$$

We shall refer to $A(t)$ and $B(t)$ as generalized switching-time functions. Substituting (2.11) in (2.10) and using the discrete Fourier transform identity

$$
\sum_{n=-\infty}^{\infty} \delta(t-n T)=\frac{1}{T} \sum_{n=-\infty}^{\infty} \mathrm{e}^{\mathrm{i} \omega_{c} n t}
$$

where $\omega_{c}=2 \pi / T$ is the carrier-wave frequency, we find

$$
\begin{aligned}
\mathcal{S} & =\frac{1}{T} \sum_{m=1}^{\infty} \frac{1}{m !}(-\mathrm{i} \omega)^{m} \sum_{n=-\infty}^{\infty} \int_{-\infty}^{\infty} \mathrm{e}^{-\mathrm{i}\left(\omega-n \omega_{c}\right) t}\left[B^{m}(t)-A^{m}(t)\right] \mathrm{d} t \\
& =\frac{(2 \pi)^{1 / 2}}{T} \sum_{m=1}^{\infty} \frac{1}{m !}(-\mathrm{i} \omega)^{m} \sum_{n=-\infty}^{\infty}\left[\hat{B}_{m}\left(\omega-n \omega_{c}\right)-\hat{A}_{m}\left(\omega-n \omega_{c}\right)\right]
\end{aligned}
$$

Here $\hat{A}_{m}$ and $\hat{B}_{m}$ are, respectively, the Fourier transforms of $A^{m}$ and $B^{m}$.

Now we use the separation of time scales between the audio signal and the carrier wave to generate a simpler approximation to this expression for $\mathcal{S}$. We begin by noting that since the signal $s(t)$ varies only slowly over a single period of the carrier wave, the switching times $\alpha_{n}$ and $\beta_{n}$ vary only slowly with $n$. Thus we assume that $A(t)$ and $B(t)$ are chosen to vary only on the relatively slow time scale of the signal and contain no components that vary on the shorter time scale of the carrier wave. (Of course, in practice this 'bandwidth limiting' is only approximate.) The upshot of this assumption is that we take into account only the term $n=0$ in the sum in (2.14); then, with this approximation,

$$
\mathcal{S}=\frac{(2 \pi)^{1 / 2}}{T} \sum_{m=1}^{\infty} \frac{1}{m !}(-1)^{m}(\mathrm{i} \omega)^{m}\left[\hat{B}_{m}(\omega)-\hat{A}_{m}(\omega)\right] .
$$

Correspondingly, from (2.9) it follows that

$$
\hat{g}(\omega)=(2 \pi)^{1 / 2} \delta(\omega)+\frac{2}{T} \sum_{m=1}^{\infty} \frac{1}{m !}(-1)^{m}(\mathrm{i} \omega)^{m-1}\left[\hat{B}_{m}(\omega)-\hat{A}_{m}(\omega)\right]
$$

and hence, upon inverting the Fourier transform, we obtain for the component of $g(t)$ in the audio spectrum (cf. $[4,5])$

$$
g_{a}(t)=1+\frac{2}{T} \sum_{m=1}^{\infty} \frac{1}{m !}(-1)^{m} \frac{\mathrm{d}^{m-1}}{\mathrm{~d} t^{m-1}}\left[B^{m}(t)-A^{m}(t)\right] .
$$


This expression applies regardless of the details of the class-D amplifier design: the differences between the various designs lie in the specific relationships between the generalized switching-time functions and the signal $s(t)$; these correspondingly result in different audio outputs $g_{a}(t)$.

2.1.1. Discussion. The infinite sum in (2.17) should, of course, be viewed with some caution, given the approximations underlying it. Even if $A(t)$ and $B(t)$ are signals whose frequency spectra lie entirely within the audio range, the powers $A^{m}$ and $B^{m}$ include successively higher frequencies in their spectra. For example, if $A$ and $B$ are pure sinusoidal signals, each with frequency $\omega$, then $A^{m}$ and $B^{m}$ involve frequencies up to $m \omega$. For sufficiently large $m$, when $\left|\omega_{c} \mp m \omega\right|$ lies in the audio range, terms with $n= \pm 1$ must be included in the sum (2.14), rendering inappropriate our assumption that only terms with $n=0$ contribute to the output audio spectrum (for larger values of $m$, additional values of $n$ also become relevant). However, in the analysis that follows we shall consider only the first few terms in (2.17), because these are sufficient to determine the principal distortion characteristics of the amplifier designs, hence for our purposes the difficulty with large values of $m$ in (2.17) is immaterial.

2.2. Alternative expression for $g_{a}(t)$. This section may be omitted by readers interested only in the class-D amplifier designs with negative feedback, since it is primarily of importance for the 'classical' design without feedback, in which case we shall see below that the equations governing $A(t)$ and $B(t)$ take the form

$$
A(t)=\bar{A}(t+A(t)), \quad B(t)=\bar{B}(t+B(t)),
$$

for some functions $\bar{A}$ and $\bar{B}$. Clearly some conditions must be imposed upon $A(t)$ and $B(t)$ in order that (2.18) defines $\bar{A}$ and $\bar{B}$ uniquely; it is sufficient that $A(t)$ and $B(t)$ should vary sufficiently slowly, i.e., $\left|A^{\prime}(t)\right|<1$ and $\left|B^{\prime}(t)\right|<1$ (cf. [5]). It then proves useful to introduce 'warped times'

$$
t_{A}=t+A(t), \quad t_{B}=t+B(t),
$$

so that

$$
t_{A}=t+\bar{A}\left(t_{A}\right), \quad t_{B}=t+\bar{B}\left(t_{B}\right) .
$$

Now to obtain a simpler expression for $g_{a}(t)$, we note from the definition of the Fourier transform (2.8) and (2.15) that $\mathcal{S}$ may be written as

$$
\mathcal{S}=\frac{1}{T} \sum_{m=1}^{\infty} \frac{(-\mathrm{i} \omega)^{m}}{m !} \int_{-\infty}^{\infty}\left[B^{m}(t)-A^{m}(t)\right] \mathrm{e}^{-\mathrm{i} \omega t} \mathrm{~d} t
$$

and hence, provided the order of the summation and integration may be interchanged,

$$
\begin{aligned}
\mathcal{S} & =\frac{1}{T} \int_{-\infty}^{\infty} \sum_{m=1}^{\infty} \frac{(-\mathrm{i} \omega)^{m}}{m !}\left[B^{m}(t)-A^{m}(t)\right] \mathrm{e}^{-\mathrm{i} \omega t} \mathrm{~d} t \\
& =\frac{1}{T} \int_{-\infty}^{\infty} \mathrm{e}^{-\mathrm{i} \omega(t+B(t))}-\mathrm{e}^{-\mathrm{i} \omega(t+A(t))} \mathrm{d} t,
\end{aligned}
$$

from which it follows that

$$
\mathrm{i} \omega \hat{g}(\omega)=\frac{2}{T} \frac{1}{(2 \pi)^{1 / 2}} \int_{-\infty}^{\infty} \mathrm{e}^{-\mathrm{i} \omega(t+B(t))}-\mathrm{e}^{-\mathrm{i} \omega(t+A(t))} \mathrm{d} t
$$




$$
\begin{aligned}
& =\frac{2}{T} \frac{1}{(2 \pi)^{1 / 2}}\left\{\int_{-\infty}^{\infty} \mathrm{e}^{-\mathrm{i} \omega t_{B}} \frac{\mathrm{d} t}{\mathrm{~d} t_{B}} \mathrm{~d} t_{B}-\int_{-\infty}^{\infty} \mathrm{e}^{-\mathrm{i} \omega t_{A}} \frac{\mathrm{d} t}{\mathrm{~d} t_{A}} \mathrm{~d} t_{A}\right\} \\
& =\frac{2}{T} \frac{\mathrm{i} \omega}{(2 \pi)^{1 / 2}}\left\{\int_{-\infty}^{\infty} \mathrm{e}^{-\mathrm{i} \omega t_{B}} t\left(t_{B}\right) \mathrm{d} t_{B}-\int_{-\infty}^{\infty} \mathrm{e}^{-\mathrm{i} \omega t_{A}} t\left(t_{A}\right) \mathrm{d} t_{A}\right\} .
\end{aligned}
$$

Thus, by inverting the Fourier transform, we find that

$$
g_{a}(t)=C_{a}+\frac{2}{T}\left[g_{A}(t)+g_{B}(t)\right]
$$

where $C_{a}$ is a constant of integration, $g_{A}\left(t_{A}\right)=-t\left(t_{A}\right)$ and $g_{B}\left(t_{B}\right)=t\left(t_{B}\right)$. From $(2.20), t\left(t_{A}\right)=t_{A}-\bar{A}\left(t_{A}\right)$ and $t\left(t_{B}\right)=t_{B}-\bar{B}\left(t_{B}\right)$. Thus

$$
g_{A}\left(t_{A}\right)=-t_{A}+\bar{A}\left(t_{A}\right), \quad g_{B}\left(t_{B}\right)=t_{B}-\bar{B}\left(t_{B}\right)
$$

or, equivalently,

$$
g_{A}(t)=-t+\bar{A}(t), \quad g_{B}(t)=t-\bar{B}(t)
$$

so that, finally, we obtain from (2.24) and (2.26)

$$
g_{a}(t)=1+\frac{2}{T}[\bar{A}(t)-\bar{B}(t)]
$$

The constant of integration $C_{a}=1$ has been fixed by noting that for zero input signal $(s(t) \equiv 0)$ it follows from $(2.2)$ that $g_{a}(t) \equiv 0$, while $\bar{A}(t) \equiv T / 4$ and $\bar{B}(t) \equiv 3 T / 4$. Thus when the problem for the generalized switching times is of the form (2.18), the audio output takes a particularly simple form, which does not appear to have been noted previously.

2.3. 'Classical' class-D amplifier. For the 'classical' class-D amplifier illustrated in Figure 2.1, we find from (2.3) and (2.12) that

$$
A(n T)=\frac{T}{4}[1+s(n T+A(n T))], \quad B(n T)=\frac{T}{4}[3-s(n T+B(n T))] .
$$

It is then straightforward to extend this definition of $A(t)$ and $B(t)$ appropriately to other times by globally mapping $n T \mapsto t$ in (2.28). Then in view of (2.18) and (2.27) it follows that

$$
g_{a}(t)=1+\frac{2}{T}\left\{\frac{T}{4}[1+s(t)]-\frac{T}{4}[3-s(t)]\right\}=s(t) .
$$

Thus (as is well known $[1,4,5]$ ) this amplifier gives no distortion to the signal (given the modeling assumptions).

Note in particular that no assumption has been made regarding the shape of the audio waveform $s(t)$ other than that its spectrum lies entirely in the audio band, well below the carrier-wave frequency; most significantly, the input signal need not be a pure sinusoid. Our derivation of this result differs somewhat from that given recently $[4,5]$, since we use (2.27) rather than apply a theorem in complex analysis due to Lagrange $[4,5]$. 


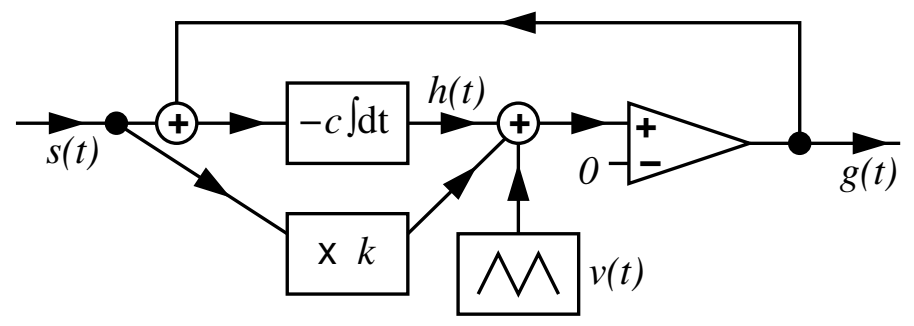

FIG. 3.1. Class-D amplifier with negative feedback. The signal $s(t)$ is fed into a device that multiplies it by a constant $k$, and also into an integrator, whose output we denote by $h(t)$. The output of the integrator and the multiplier are summed, together with a high-frequency triangular carrier wave $v(t)$, and input to the noninverting input of a comparator whose inverting input is grounded. The output of the comparator is $g(t)$.

3. Class-D amplifier with negative feedback. With negative feedback, the basic class-D amplifier design is illustrated in Figure 3.1.

In analysing the amplifier design, we find it convenient to introduce $f(t)$, the integral of the input signal, defined by $f^{\prime}(t)=s(t)$; the constant of integration in determining $f(t)$ uniquely is not important in what follows. The triangular carrier wave $v(t)$ again satisfies $(2.1)$ and the periodicity condition $v(t+T)=v(t)$ for all $t$. The output $g(t)$ of the comparator now satisfies

$$
g(t)= \begin{cases}+1 & \text { if } h(t)+k s(t)+v(t)>0 \\ -1 & \text { if } h(t)+k s(t)+v(t)<0\end{cases}
$$

Finally, the integrator output is given by

$$
h(t)=-c \int^{t} g(\tau)+s(\tau) \mathrm{d} \tau .
$$

The time constant $c$ is such that $c T=O(1)$. Since $-1<s(t)<1, h(t)$ alternately increases and decreases, as $g(t)$ is, respectively, negative and positive. The relationships between $v(t), g(t)$ and $h(t)$ are illustrated in Figure 3.2. We note that for illustrative purposes the figure shows $h(t)$ as a piecewise linear function of time, which is appropriate only for a constant input signal; otherwise $h(t)$ has a slight nonlinearity.

3.1. Analysis of the model. We analyze the model by first constructing a system of nonlinear implicit difference equations for the switching times $\alpha_{n}$ and $\beta_{n}$. To do so, we consider a time interval $n T<t<(n+1) T$. Referring to the waveform in Figure 3.2, we see that at the start and end of this interval, $h(t)$ is decreasing; in between, $h(t)$ is increasing. We define three subintervals:

$$
\begin{array}{lll}
\text { I: } & n T<t<n T+\alpha_{n} & h^{\prime}(t)<0[g(t)=1] ; \\
\text { II: } & n T+\alpha_{n}<t<n T+\beta_{n} & h^{\prime}(t)>0[g(t)=-1] ; \\
\text { III: } & n T+\beta_{n}<t<(n+1) T & h^{\prime}(t)<0[g(t)=1],
\end{array}
$$

and consider each in turn.

Subinterval I. By integrating (3.2) we find

$$
h(t)=h(n T)-c[f(t)-f(n T)]-c(t-n T) .
$$




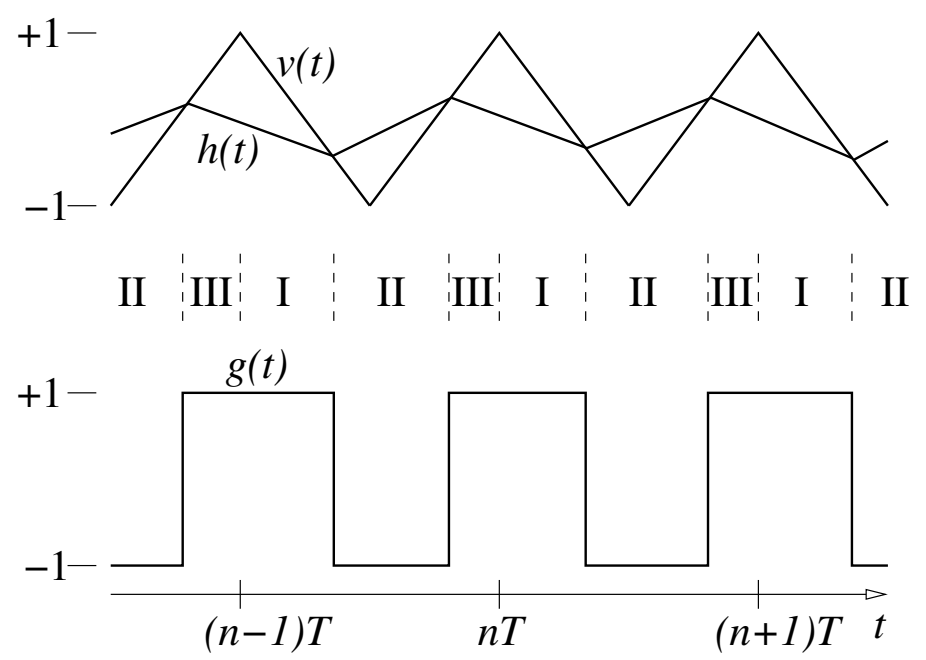

FIG. 3.2. Diagram showing relationships between $v(t), g(t)$ and $h(t)$ for the class-D amplifier with negative feedback. Note that, although $h(t)$ is here drawn as piecewise linear, it is in fact nonlinear for any nontrivial input signal $s(t)$. The subintervals I, II and III are indicated, as defined in (3.3).

According to (3.1), the value of $\alpha_{n}$ is defined by

$$
h\left(n T+\alpha_{n}\right)+k s\left(n T+\alpha_{n}\right)+v\left(n T+\alpha_{n}\right)=0,
$$

that is,

$$
h(n T)-c\left[f\left(n T+\alpha_{n}\right)-f(n T)\right]-c \alpha_{n}+k s\left(n T+\alpha_{n}\right)+1-\frac{4 \alpha_{n}}{T}=0 .
$$

Subinterval II. By integrating (3.2) and enforcing continuity of $h(t)$ at time $t=n T+\alpha_{n}$, we find

$$
h(t)=h(n T)-c[f(t)-f(n T)]-c \alpha_{n}+c\left(t-n T-\alpha_{n}\right) .
$$

From (3.1), the value of $\beta_{n}$ is defined by

$$
h\left(n T+\beta_{n}\right)+k s\left(n T+\beta_{n}\right)+v\left(n T+\beta_{n}\right)=0,
$$

that is,

$\left(3.9 \hbar(n T)-c\left[f\left(n T+\beta_{n}\right)-f(n T)\right]+c\left(\beta_{n}-2 \alpha_{n}\right)+k s\left(n T+\beta_{n}\right)-3+\frac{4 \beta_{n}}{T}=0\right.$.

Subinterval III. By integrating (3.2) and enforcing continuity of $h(t)$ at time $t=n T+\beta_{n}$, we find

$$
h(t)=h(n T)-c[f(t)-f(n T)]+c\left(\beta_{n}-2 \alpha_{n}\right)-c\left(t-n T-\beta_{n}\right) .
$$

For the remaining analysis, we note that at the end of this subinterval

$$
h((n+1) T)=h(n T)-c[f((n+1) T)-f(n T)]+c\left(2 \beta_{n}-2 \alpha_{n}-T\right) .
$$




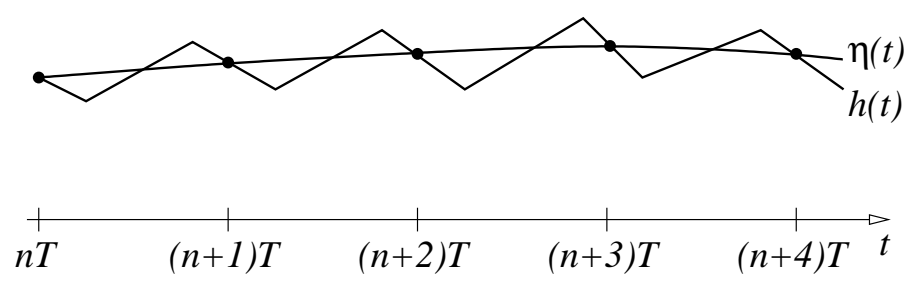

FiG. 3.3. Diagram showing relationship between $h(t)$ and $\eta(t)$. The two functions agree at times $n T$ (for integers $n$ ), but $h(t)$ varies significantly at intermediate times, whereas $\eta$ is only slowly varying.

3.2. Solution of the governing equations. Our goal is to use (2.17) to determine the audio output of the amplifier. To do so we first need to determine the switching times of $g(t)$. Given a signal $s(t)$, we determine these switching times, together with the values of $h(n T)$, from the coupled equations (3.6), (3.9) and (3.11), which are, so far, exact.

We first use (2.12) to substitute the generalized switching-time functions for $\alpha_{n}$ and $\beta_{n}$; the result is a system of three equations involving $A(n T), B(n T)$ and $h(n T)$. These equations are readily extended to intermediate times by mapping $n T \mapsto t$ :

(3.13) $\left(\frac{4}{T}+c\right) B(t)=3-k s(t+B(t))-\eta(t)+c[f(t+B(t))-f(t)]+2 c A(t)$,

$$
\eta(t+T)=\eta(t)-c[f(t+T)-f(t)]+c[2 B(t)-2 A(t)-T] .
$$

Since the functions $A(t)$ and $B(t)$ vary only on the time scale of the audio signal and not on that of the carrier wave, the function $h(t)$ is replaced in these expressions by a slowly varying function $\eta(t)$ such that

$$
\eta(n T)=h(n T)
$$

(see Figure 3.3).

Given the mild restrictions on the form of the input signal $s(t)$ and its first integral $f(t)$, it seems unlikely that a general solution can be found to the coupled nonlinear equations (3.12)-(3.14). Furthermore, it seems unlikely that any solution will be unique, although we are unable to demonstrate nonuniqueness for the system of three equations as posed. (A suggestion of nonuniqueness comes from the following thought experiment. Suppose $A(t)$ and $B(t)$ are known and independent of $\eta(t)$. Then the solution $\eta(t)$ to (3.14) is unique only up to the addition of a function of period $T$ [3]. Note that here the nonuniqueness involves high-frequency components only.)

We find that we are able to construct a solution to (3.12)-(3.14) with frequency spectrum in the audio range, as follows. The derivation is admittedly rather informal. We introduce the small parameter $\epsilon=\omega_{\text {typ }} / \omega_{c} \ll 1$, where $\omega_{\text {typ }}$ is a typical audio frequency component of the input. Then we note that (3.12)-(3.14) relate to variations in $s, A, B$ and $\eta$ on a time scale $t=O(T)$, and that such variations satisfy

$$
\frac{\mathrm{d}^{n}}{\mathrm{~d} t^{n}}=O\left(\epsilon^{n}\right)
$$


We then expand $A(t), B(t)$ and $\eta(t)$ as series

$$
A(t)=\sum_{m=0}^{\infty} A_{m}(t), \quad B(t)=\sum_{m=0}^{\infty} B_{m}(t), \quad \eta(t)=\sum_{m=0}^{\infty} \eta_{m}(t),
$$

where the terms in (3.16) satisfy

$$
A_{m}, B_{m}, \eta_{m}=O\left(\epsilon^{m}\right) .
$$

For all functions in (3.12)-(3.14) not evaluated at time $t$, we make use of Taylor expansions such as

$$
\eta(t+T)=\sum_{n=0}^{\infty} T^{n} \frac{\eta^{(n)}(t)}{n !}
$$

to write them in terms of functions and derivatives evaluated at time $t$.

As an aside, we note that an alternative mathematical solution may possibly be developed using the calculus of finite differences [3], where problems such as (3.14), of the form $u(t+T)-u(t)=U(t)$, are examples of the so-called 'summation problem'. However, the added complication of (3.12) and (3.13) makes a complete explicit solution unlikely. We note, however, that (3.14) has the exact formal solution

$$
\eta(t)=-c f(t)+c \sum_{n=1}^{\infty}[2 B(t-n T)-2 A(t-n T)-T] .
$$

Some of the key terms in the various expansions are

$$
\begin{aligned}
f(t+T)-f(t)= & T s(t)+\frac{1}{2} T^{2} s^{\prime}(t)+\frac{1}{6} T^{3} s^{\prime \prime}(t)+O\left(\epsilon^{3}\right) \\
\eta(t+T)-\eta(t)= & T \eta_{0}^{\prime}(t)+\left[T \eta_{1}^{\prime}(t)+\frac{1}{2} T^{2} \eta_{0}^{\prime \prime}(t)\right]+O\left(\epsilon^{3}\right) \\
s(t+A(t))= & s(t)+A_{0}(t) s^{\prime}(t)+\left[\frac{1}{2} A_{0}^{2}(t) s^{\prime \prime}(t)+A_{1}(t) s^{\prime}(t)\right]+O\left(\epsilon^{3}\right) \\
s(t+B(t))= & s(t)+B_{0}(t) s^{\prime}(t)+\left[\frac{1}{2} B_{0}^{2}(t) s^{\prime \prime}(t)+B_{1}(t) s^{\prime}(t)\right]+O\left(\epsilon^{3}\right) \\
f(t+A(t))-f(t)= & A_{0}(t) s(t)+\left[\frac{1}{2} A_{0}^{2}(t) s^{\prime}(t)+A_{1}(t) s(t)\right] \\
& +\left[\frac{1}{6} A_{0}^{3}(t) s^{\prime \prime}(t)+A_{0}(t) A_{1}(t) s^{\prime}(t)+A_{2}(t) s(t)\right]+O\left(\epsilon^{3}\right) \\
f(t+B(t))-f(t)= & B_{0}(t) s(t)+\left[\frac{1}{2} B_{0}^{2}(t) s^{\prime}(t)+B_{1}(t) s(t)\right] \\
& +\left[\frac{1}{6} B_{0}^{3}(t) s^{\prime \prime}(t)+B_{0}(t) B_{1}(t) s^{\prime}(t)+B_{2}(t) s(t)\right]+O\left(\epsilon^{3}\right) .
\end{aligned}
$$

In view of (2.17), we also have the expansion

$$
\begin{aligned}
g_{a}(t)= & -\frac{2}{T}\left(B_{0}-A_{0}\right) \\
& +\frac{1}{T}\left[\left(B_{0}^{2}-A_{0}^{2}\right)^{\prime}-2\left(B_{1}-A_{1}\right)\right] \\
& +\frac{1}{T}\left[-\frac{1}{3}\left(B_{0}^{3}-A_{0}^{3}\right)^{\prime \prime}+2\left(B_{0} B_{1}-A_{0} A_{1}\right)^{\prime}-2\left(B_{2}-A_{2}\right)\right]+O\left(\epsilon^{3}\right)
\end{aligned}
$$

for the audio output. With obvious notation, we write this as

$$
g_{a}(t)=g_{0}(t)+g_{1}(t)+g_{2}(t)+O\left(\epsilon^{3}\right) .
$$

Let us now consider the problem (3.12)-(3.14) at successive powers of $\epsilon$, starting with terms of $O\left(\epsilon^{0}\right)$. In doing so, it proves useful to note that upon adding (3.12) 
to (3.13) we eliminate the unknown function $\eta(t)$ and arrive at an equation that at $O\left(\epsilon^{n}\right)$ takes the form

$$
\{4-c T[1-s(t)]\} A_{n}(t)+\{4+c T[1-s(t)]\} B_{n}(t)=P_{n}(t),
$$

where $P_{n}(t)$ is known in terms of quantities calculated at previous stages in the calculation. Furthermore, (3.14) can be written in the form

$$
B_{n}(t)-A_{n}(t)=Q_{n}(t),
$$

where again $Q_{n}(t)$ comprises known quantities. This system is readily solved to give

$$
\begin{aligned}
& A_{n}(t)=\frac{1}{8}\left\{P_{n}(t)-[4+c T(1-s(t))] Q_{n}(t)\right\}, \\
& B_{n}(t)=\frac{1}{8}\left\{P_{n}(t)+[4-c T(1-s(t))] Q_{n}(t)\right\} .
\end{aligned}
$$

At $O\left(\epsilon^{0}\right)$, we find $P_{0}=4 T$ and $Q_{0}=\frac{1}{2} T(1+s(t))$. Correspondingly,

$$
\begin{aligned}
& A_{0}=\frac{1}{16} T(1-s(t))[4-c T(1+s(t))] \\
& B_{0}=\frac{1}{2} T+\frac{1}{16} T(1+s(t))[4-c T(1-s(t))],
\end{aligned}
$$

so the switching times approximately satisfy

$$
\begin{aligned}
\alpha_{n} & =\frac{1}{16} T(1-s(n T))[4-c T(1+s(n T))] \\
\beta_{n} & =\frac{1}{2} T+\frac{1}{16} T(1+s(n T))[4-c T(1-s(n T))] .
\end{aligned}
$$

Of most significance is the result, which now follows from (3.24), (3.25) and (3.18), that

$$
g_{0}(t)=-s(t) .
$$

Thus to this order there is no distortion from signal to output, apart from a sign change, which is unimportant for audio applications. However, in contrast to the 'classical' design, the next orders in the expansion of the audio output reveal distortion inherent in the nonlinear-feedback design. (The minus sign in (3.28) is an artifact of applying the triangular wave input to the noninverting input of the comparator; if it is instead applied to the inverting input then there is no sign change to the output.)

3.3. Amplifier output. The next steps in the calculation are algebraically cumbersome and shed little further light on the problem, so the full details are not presented here. Our primary interest lies in the audio output, and this turns out to be

$$
\begin{aligned}
g_{a}(t)= & -s(t)+\frac{1+k}{c} s^{\prime}(t) \\
& -\frac{1}{48 c^{2}}\left\{\left[48(1+k)-c^{2} T^{2}\right] s(t)-c^{2} T^{2} s^{3}(t)\right\}^{\prime \prime}+O\left(\epsilon^{3}\right) .
\end{aligned}
$$

Note that there arises a nonlinear (cubic) distortion term; this term is to leading order independent of $k$, so it cannot be removed by any choice of this parameter. This nonlinear term represents the 'intrinsic' distortion of class-D amplifiers with negative feedback to which we have alluded above.

The linear terms in (3.29) also represent a form of distortion to the signal since they affect different frequency components to different extents. This distortion can 
be removed by making an appropriate choice of $k$ such that the linear terms in (3.29) form the beginnings of a Taylor series for a slightly delayed signal $-s(t-(1+k) / c)$. It is readily determined that the appropriate value of $k$ satisfies

$$
k^{2}=1-\frac{1}{24} c^{2} T^{2}
$$

correspondingly, the audio output is then given by

$$
g_{a}(t)=-s(t-(1+k) / c)+\frac{1}{48} T^{2}\left(s^{3}(t)\right)^{\prime \prime}+O\left(\epsilon^{3}\right) .
$$

Note that the delay to the output indicated here is independent of signal amplitude and frequency, and thus is entirely benign. Furthermore, $(1+k) / c$ is a time of the order of the carrier-wave period, so the delay to the audio signal is slight. However, the nonlinear distortion term remains.

For the specific case of a sinusoidal input signal $s(t)=s_{0} \sin \omega t,(3.29)$ becomes

$g_{a}(t)=-s_{0} \sin \omega t+(1+k) \mu s_{0} \cos \omega t$

$$
+\frac{\mu^{2}}{192}\left\{\left[192(1+k)-\left(4+3 s_{0}^{2}\right) c^{2} T^{2}\right] s_{0} \sin \omega t+9 c^{2} T^{2} s_{0}^{3} \sin 3 \omega t\right\}+O\left(\mu^{3}\right),
$$

where $\mu=O(\epsilon)$ : specifically

$$
\mu=\frac{\omega T}{c T} \ll 1
$$

We note from (3.32) that the intrinsic nonlinear distortion manifests itself through both a nonlinear influence on the amplitude of the fundamental, and the presence of a third-harmonic term.

3.4. Alternative expression for $g_{a}(t)$. An alternative expression for the audio output may be derived as follows. First we note that the switching times for $g(t)$ satisfy

$$
\begin{aligned}
\alpha_{n} & =\frac{T}{4}\left[1+h\left(n T+\alpha_{n}\right)+k s\left(n T+\alpha_{n}\right)\right], \\
\beta_{n} & =\frac{T}{4}\left[3-h\left(n T+\beta_{n}\right)-k s\left(n T+\beta_{n}\right)\right] .
\end{aligned}
$$

If we introduce two new slowly varying functions $\eta(t ; \alpha)$ and $\eta(t ; \beta)$ defined so that

$$
\eta\left(n T+\alpha_{n} ; \alpha\right)=h\left(n T+\alpha_{n}\right), \quad \eta\left(n T+\beta_{n} ; \beta\right)=h\left(n T+\beta_{n}\right),
$$

then it follows from (2.29) that

$$
g_{a}(t)=\frac{1}{2}[\eta(t ; \alpha)+\eta(t ; \beta)]+k s(t) .
$$

Although this expression does not yield a useful explicit exact formula for $g_{a}(t)$, it does provide an alternative means of calculating $g_{a}(t)$. This in turn gives us an independent check on our results, which we have used to verify expressions such as (3.29).

Having highlighted the third-harmonic distortion generated by the simplest classD amplifier design with negative feedback, we proceed to describe some remedies. 


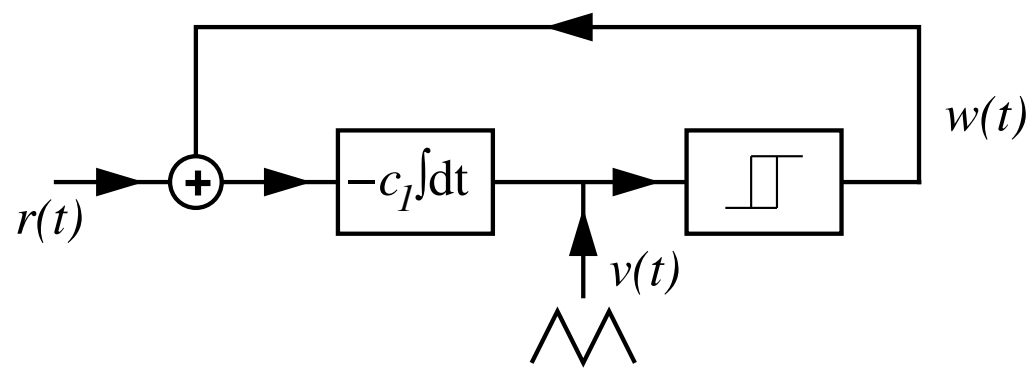

FIG. 4.1. Circuit diagram for modulation of the carrier wave. The modulation function $r(t)$ is input to an integrator, whose output $v(t)$ is input to a hysteresis loop. The output $w(t)$ of the last device takes the value +1 once its input has reached the value +1 ; thereafter $w(t)$ remains at +1 , until the input $v(t)$ falls to -1 , from which point onwards $w(t)=-1$, until the input $v(t)$ reaches +1 again, and so on.

4. Modulation of the carrier wave. We now examine one means of eliminating the 'intrinsic distortion' term in (3.29). The key to the technique is to modulate the carrier wave in such a way that the switching times of $g(t)$ are slightly altered in an appropriate fashion to counter the distortion. This technique is used successfully for distortion reduction in amplifiers manufactured by Halcro Pty Ltd (www . halcro.com).

We suppose that the carrier wave is modulated by a slowly varying input signal $r(t)$, where $r(t)=e^{\prime}(t)$, for some function $e(t)$; since $r(t)$ involves a first derivative, it is taken to be $O(\epsilon)$, and the modulation of the carrier wave correspondingly slight. The modulation circuit is illustrated in Figure 4.1; the full amplifier circuit is caricatured in Figure 4.2. Now the carrier wave $v(t)$ is governed by

$$
v(t)=-c_{1} \int^{t} w(\tau)+r(\tau) \mathrm{d} \tau,
$$

where $c_{1}$ is a time constant associated with the integrator in the modulation circuit (see Figure 4.1), and is no longer quite piecewise linear. The signal $w(t)$ is a square wave of variable duty cycle, taking the values $w(t)= \pm 1$, depending on the carrier wave $v(t)$ as follows. First, suppose that $w=-1$; then $v$ is increasing. When $v$ reaches $+1, w$ changes to +1 and $v$ starts to decrease. Once $v$ has decreased to -1 , $w$ changes to -1 ; then $v$ starts to increase once more, until it reaches +1 again, at which point the cycle starts over.

4.1. Analysis of the model. Suppose that $w(t)$ changes to the value $w=1$ at times $t=T_{n}$, and changes to the value $w=-1$ at times $t=U_{n}$. Then for $T_{n}<t<U_{n}, w(t)=1$ and hence

$$
v(t)=1-c_{1}\left[e(t)-e\left(T_{n}\right)\right]-c_{1}\left(t-T_{n}\right) .
$$

For $U_{n}<t<T_{n+1}, w(t)=-1$ and

$$
v(t)=-1-c_{1}\left[e(t)-e\left(U_{n}\right)\right]+c_{1}\left(t-U_{n}\right) .
$$

The time constant $c_{1}$ (determined below) is such that $c_{1} T=O(1)$. Constants of integration have been chosen so that each of these expressions gives the correct value for $v(t)$ at the start of each time interval. Imposing the appropriate value for $v(t)$ at the end of each time interval then gives the two conditions

$$
\begin{aligned}
c_{1}\left[e\left(U_{n}\right)-e\left(T_{n}\right)\right]+c_{1}\left(U_{n}-T_{n}\right) & =2 \\
-c_{1}\left[e\left(T_{n+1}\right)-e\left(U_{n}\right)\right]+c_{1}\left(T_{n+1}-U_{n}\right) & =2 .
\end{aligned}
$$




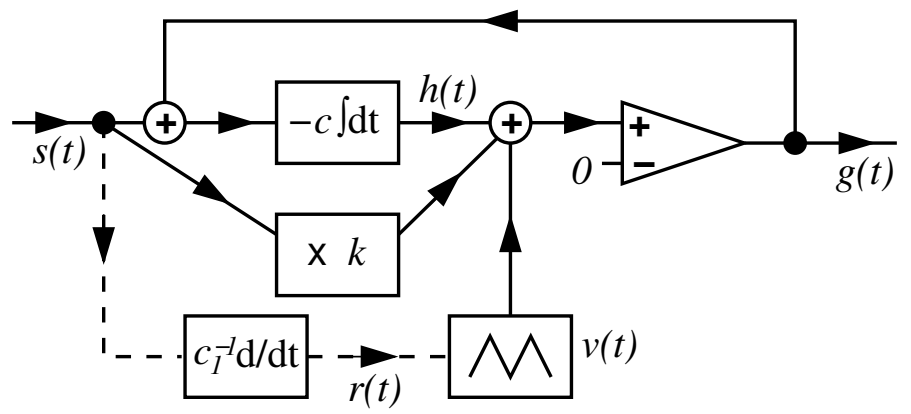

FIG. 4.2. Class-D amplifier with negative feedback and modulation of the carrier-wave symmetry. Note that the entire circuitry of Figure 4.1 is represented by the single box marked ' $v(t)$ '. The appropriate modulation signal $r(t)=c_{1}^{-1} s^{\prime}(t)$ is indicated on the diagram, as determined in Section 4.1.2.

For the special case in which $r(t) \equiv r_{0}$ is constant, the carrier wave $v(t)$ is timeperiodic, with period of oscillation

$$
T=\frac{4}{\left(1-r_{0}^{2}\right) c_{1}} \sim \frac{4}{c_{1}}\left(1+r_{0}^{2}\right) .
$$

Furthermore, in this case

$$
U_{n}-T_{n}=\frac{2}{\left(1+r_{0}\right) c_{1}} \sim \frac{2}{c_{1}}\left(1-r_{0}\right)
$$

Note that $T$ is in general increased by the presence of a nonzero modulation signal (i.e., the frequency of the carrier wave is reduced). In what follows, we shall require corrections to the carrier-wave due to modulation only up to $O(\epsilon)$, and thus, since $r^{2}=O\left(\epsilon^{2}\right)$, we may take $T$ as fixed. Then the time constant $c_{1}$ must be chosen so that

$$
c_{1}=\frac{4}{T}
$$

With this approximation, it turns out that we may consistently calculate terms in $g_{a}(t)$ up to $O\left(\epsilon^{2}\right)$, which is sufficient to determine the effects of carrier-wave modulation on the amplifier's distortion characteristics.

If we write the times at which the slope of the triangular wave changes as

$$
T_{n}=n T+a_{n}, \quad U_{n}=n T+b_{n},
$$

where $0<a_{n}<b_{n}<T$, then these times are now governed by

$$
\begin{aligned}
0= & h(n T)-c\left[f\left(n T+\alpha_{n}\right)-f(n T)\right]-c \alpha_{n}+k s\left(n T+\alpha_{n}\right) \\
& +1-c_{1}\left[e\left(n T+\alpha_{n}\right)-e\left(n T+a_{n}\right)\right]-c_{1}\left(\alpha_{n}-a_{n}\right) \\
0= & h(n T)-c\left[f\left(n T+\beta_{n}\right)-f(n T)\right]+c\left(\beta_{n}-2 \alpha_{n}\right)+k s\left(n T+\beta_{n}\right) \\
& -1-c_{1}\left[e\left(n T+\beta_{n}\right)-e\left(n T+b_{n}\right)\right]+c_{1}\left(\beta_{n}-b_{n}\right),
\end{aligned}
$$

rather than by (3.6) and (3.9). As in the absence of modulation, we have (3.14). 
The solution technique is just as with no modulation. Again we seek slowly varying generalized switching times $A(t)$ and $B(t)$ of the output $g(t)$, but now we need two further slowly varying functions, $C(t)$ and $D(t)$, such that

$$
C(n T)=a_{n}, \quad D(n T)=b_{n} .
$$

The equations to be solved arise from (3.14), (4.4), (4.5), (4.8) and (4.9), and are

$$
\begin{aligned}
c_{1}[e(t+D(t))-e(t+C(t))]+c_{1}(D(t)-C(t)) & =2 \\
-c_{1}[e(t+T+C(t+T))-e(t+D(t))]+c_{1}(T+C(t+T)-D(t)) & =2 \\
\eta(t)-c[f(t+A(t))-f(t)]-c A(t)+k s(t+A(t)) & \\
+1-c_{1}[e(t+A(t))-e(t+C(t))]-c_{1}(A(t)-C(t)) & =0 \\
\eta(t)-c[f(t+B(t))-f(t)]+c(B(t)-2 A(t))+k s(t+B(t)) & \\
-1-c_{1}[e(t+B(t))-e(t+D(t))]+c_{1}(B(t)-D(t)) & =0 \\
\eta(t+T)-\eta(t)+c[f(t+T)-f(t)]-c[2 B(t)-2 A(t)-T] & =0
\end{aligned}
$$

As with the simpler case of an unmodulated carrier wave, we expand all unknown functions (here $A, B, C, D$ and $\eta$ ) as series, as in (3.16), and solve in succession for the terms in these series at the first few orders.

4.1.1. Discussion. When expanded, (4.11) and (4.12) each yield at $O(1)$ and at $O(\epsilon)$ identical equations, of the forms

$$
D_{0}(t)-C_{0}(t)=\frac{1}{2} T, \quad D_{1}(t)-C_{1}(t)=-\frac{1}{2} \operatorname{Tr}(t),
$$

respectively. The fact that only the difference between the times $C$ and $D$ may be determined partly reflects an arbitrariness in the time origin for the circuit that generates the carrier wave. However, if we continue to the next order we find that the two equations for $D_{2}(t)-C_{2}(t)$ are in fact inconsistent, reflecting the more serious limitation imposed upon the analysis by our assumption that the mean carrier-wave period is unaltered by the modulation. Fortunately, a consistent calculation of $C$ and $D$ up to terms $C_{1}$ and $D_{1}$ proves sufficient to determine the audio output of the amplifier up to $g_{2}$, which allows us to calculate the elimination of the distortion.

4.1.2. Elimination of the distortion. We find the audio output to be

$$
\begin{aligned}
& g_{a}(t)=-s(t)+\frac{1+k}{c} s^{\prime}(t) \\
& 4.17) \quad-\frac{1}{3 c_{1}^{2} c^{2}}\left\{3 c_{1} c^{2}\left(r s^{2}\right)^{\prime}+\left[3(1+k) c_{1}^{2}-c^{2}\right] s^{\prime \prime}-3 c_{1} c^{2} r^{\prime}-c^{2}\left(s^{3}\right)^{\prime \prime}\right\}+O\left(\epsilon^{3}\right),
\end{aligned}
$$

where $c_{1}$ is given by (4.6). There are two nonlinear distortion terms in this expression, proportional to $\left(r s^{2}\right)^{\prime}$ and $\left(s^{3}\right)^{\prime \prime}$. If we set $r=\nu s^{\prime}(t)$, we may eliminate both of them by choosing $\nu=1 / c_{1}=T / 4$. Then

$$
g_{a}(t)=-s(t)+\frac{1+k}{c} s^{\prime}(t)-\frac{1}{12 c^{2}}\left[12(1+k)-c^{2} T^{2}\right] s^{\prime \prime}(t)+O\left(\epsilon^{3}\right) .
$$

Thus all nonlinear distortion is removed, at least to the order calculated. A key result of the present analysis is that the appropriate modulation of the carrier wave is through a derivative of the input signal; this is in fact the method used in practice.

Now if we choose $k^{2}=1-\frac{1}{6} c^{2} T^{2}$, then the audio output is of the form

$$
g_{a}(t)=-s(t-(1+k) / c)+O\left(\epsilon^{3}\right),
$$

and, to the order calculated, there is no distortion beyond a slight delay to the output. 


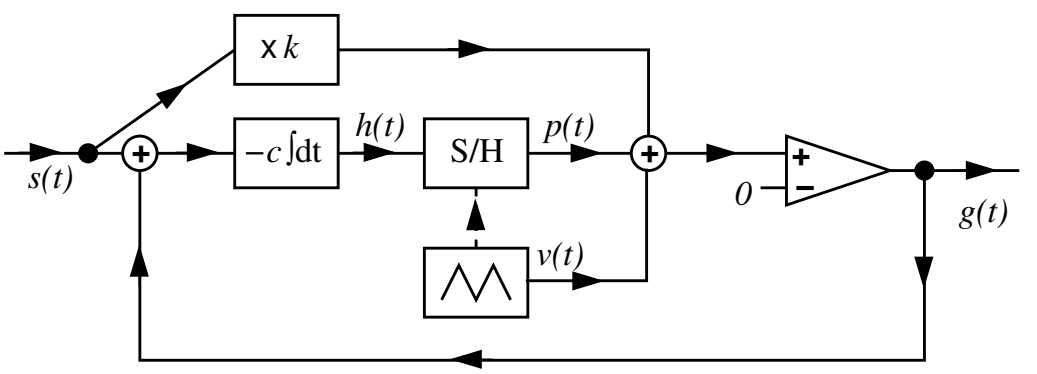

FIG. 5.1. 'Sample-and-hold' class-D amplifier with negative feedback. The sample-and-hold (' $S / H$ ') device is synchronized with the carrier-wave generator and gives an output $p(t)$ equal to its input $h(t)$ sampled at times $t=n T$ and $t=\left(n+\frac{1}{2}\right) T$. Thus for $n T \leq t<\left(n+\frac{1}{2}\right) T, p(t)=h(n T)$; correspondingly, for $\left(n+\frac{1}{2}\right) T \leq t<(n+1) T, p(t)=h\left(\left(n+\frac{1}{2}\right) T\right)$.

5. 'Sample-and-hold' class-D amplifier with negative feedback. Modulation of the carrier wave is not the only means by which the leading-order nonlinear distortion can be removed from class-D amplifiers with negative feedback. We now describe an alternative means of eliminating this distortion, without carrier-wave modulation. This alternative amplifier design has also been constructed, in prototype, by Halcro Pty Ltd, and is illustrated in Figure 5.1. Now there is no modulation of the carrier-wave symmetry.

Now the output of the integrator inputs to a sample-and-hold device, which samples $h(t)$ at times $t=n T$ and $\left(n+\frac{1}{2}\right) T$; its output $p(t)$ is then a piecewiseconstant function. For $n T \leq t<\left(n+\frac{1}{2}\right) T, p(t)$ takes the value $h(n T)$, while for $\left(n+\frac{1}{2}\right) T \leq t<(n+1) T, p(t)$ takes the value $h\left(\left(n+\frac{1}{2}\right) T\right)$. Aside from this new feature, most details of the model remain essentially unchanged. The triangular wave $v(t)$ again satisfies $(2.1)$ and $v(t+T)=v(t)$ for all $t$. The output $g(t)$ of the comparator is now +1 if $p(t)+k s(t)+v(t)>0$ and -1 if the inequality is reversed; the switching times thus satisfy

$p\left(n T+\alpha_{n}\right)+k s\left(n T+\alpha_{n}\right)+v\left(n T+\alpha_{n}\right)=0, \quad p\left(n T+\beta_{n}\right)+k s\left(n T+\beta_{n}\right)+v\left(n T+\beta_{n}\right)=0$.

The integrator output $h(t)$ is again given by (3.2). In any interval $n T<t<(n+1) T$, there are three subintervals, as in (3.3); we describe these below. The analysis is somewhat simplified by the sampling.

Subinterval I. By integrating (3.2) we find that $h(t)$ is again given by (3.4), but now the value of $\alpha_{n}$ is defined by

$$
p\left(n T+\alpha_{n}\right)+k s\left(n T+\alpha_{n}\right)+v\left(n T+\alpha_{n}\right)=0,
$$

that is,

$$
h(n T)+k s\left(n T+\alpha_{n}\right)+1-\frac{4 \alpha_{n}}{T}=0 .
$$

Subinterval II. By integrating (3.2) we again find (3.7) for $h(t)$; the value of $\beta_{n}$ is defined by

$$
p\left(n T+\beta_{n}\right)+k s\left(n T+\beta_{n}\right)+v\left(n T+\beta_{n}\right)=0,
$$

that is,

$$
h\left(\left(n+\frac{1}{2}\right) T\right)+k s\left(n T+\beta_{n}\right)-3+\frac{4 \beta_{n}}{T}=0 .
$$


Subinterval III. By integrating (3.2) we find (3.10) for $h(t)$; it follows that $h((n+1) T)$ is again given by $(3.11)$.

5.1. Solution of the governing equations. The three governing equations are now

$$
\begin{aligned}
\frac{4}{T} \alpha_{n} & =1+k s\left(n T+\alpha_{n}\right)+h(n T), \\
\frac{4}{T} \beta_{n} & =3-k s\left(n T+\beta_{n}\right)-h\left(\left(n+\frac{1}{2}\right) T\right), \\
h((n+1) T) & =h(n T)-c[f((n+1) T)-f(n T)]+c\left(2 \beta_{n}-2 \alpha_{n}-T\right) .
\end{aligned}
$$

Furthermore, by considering Subinterval II, we find that

$$
h\left(\left(n+\frac{1}{2}\right) T\right)=h(n T)-c\left[f\left(\left(n+\frac{1}{2}\right) T\right)-f(n T)\right]+c\left(\frac{T}{2}-2 \alpha_{n}\right) .
$$

As above, we introduce the slowly varying functions $A(t), B(t)$ and $\eta(t)$, which now satisfy

$$
\begin{aligned}
\frac{4}{T} A(t) & =1+k s(t+A(t))+\eta(t), \\
\frac{4}{T} B(t) & =3-k s(t+B(t))-\eta(t)+c[f(t+T / 2)-f(t)]-c[T / 2-2 A(t)], \\
\eta(t+T) & =\eta(t)-c[f(t+T)-f(t)]+c[2 B(t)-2 A(t)-T],
\end{aligned}
$$

and solve these equations at successive orders in $\epsilon$. The audio output is eventually found to be

$$
\begin{gathered}
g_{a}(t)=-s(t)+\frac{1+k}{c} s^{\prime}(t) \\
\quad+\frac{1}{96 c^{2}(4-c T)}\left\{\left[c^{3} T^{3}-(28+24 k) c^{2} T^{2}+192(1+k) c T-384(1+k)\right] s(t)\right. \\
\left.\quad+[c T-4(1+2 k)] c^{2} T^{2} s^{3}(t)\right\}^{\prime \prime}+O\left(\epsilon^{3}\right) .
\end{gathered}
$$

Now the nonlinear distortion term proportional to $\left(s^{3}\right)^{\prime \prime}$ may be removed from this expression by choosing

$$
k=-\frac{1}{2}+\frac{1}{8} c T,
$$

in which case

$$
g_{a}(t)=-s(t)+\frac{4+c T}{8 c} s^{\prime}(t)-\frac{24-c^{2} T^{2}}{48 c^{2}} s^{\prime \prime}(t)+O\left(\epsilon^{3}\right) .
$$

Once the nonlinear distortion term has been so removed, we may similarly remove linear distortion, so that the audio output only suffers a slight delay, i.e.,

$$
g_{a}(t)=-s\left(t-\frac{1}{8}(4+c T) / c\right)+O\left(\epsilon^{3}\right),
$$

by choosing $c T=12(2 \sqrt{3}-1) / 11 \approx 2.6881$. 
5.2. Alternative means of sampling. Suppose now that the sampling is carried out only at times $t=n T$ (i.e., not also at times $\left.t=\left(n+\frac{1}{2}\right) T\right)$. Then the equations to be solved for $A(t), B(t)$ and $\eta(t)$ are somewhat simplified:

$$
\begin{aligned}
\frac{4}{T} A(t) & =1+k s(t+A(t))+\eta(t), \\
\frac{4}{T} B(t) & =3-k s(t+B(t))-\eta(t), \\
\eta(t+T)-\eta(t) & =-c[f(t+T)-f(t)]+c[2 B(t)-2 A(t)-T] .
\end{aligned}
$$

The audio output is, correspondingly, found to be

$$
\begin{aligned}
g_{a}(t)=-s(t)+ & \frac{1+k}{c} s^{\prime}(t)+\frac{1}{96 c^{2}}\left\{-(2 k+1) c^{2} T^{2}\left(3 s^{2}(t)+s^{3}(t)\right)^{\prime \prime}\right. \\
& \left.+\left[-c^{2} T^{2}-6(1+k) c^{2} T^{2}+48(1+k) c T-96(1+k)\right] s^{\prime \prime}(t)\right\} .
\end{aligned}
$$

In view of the asymmetrical sampling, there are now second and third harmonics at $O\left(\epsilon^{2}\right)$, but these can simultaneously be removed by choosing

$$
k=-\frac{1}{2} .
$$

If this choice is made, then $g_{a}(t)$ becomes

$$
g_{a}(t)=-s(t)+\frac{1}{2 c} s^{\prime}(t)-\frac{1}{24 c^{2}}\left[12-6 c T+c^{2} T^{2}\right] s^{\prime \prime}(t)+O\left(\epsilon^{3}\right) .
$$

This is a delayed version of the original signal $\left(g_{a}(t)=-s\left(t-\frac{1}{2} c\right)+O\left(\epsilon^{3}\right)\right)$ if the choice $c T=3$ is made.

6. A new class-D amplifier, with reduced distortion. We now describe a third modification to the standard negative-feedback class-D amplifier, which eliminates the intrinsic distortion at $O\left(\epsilon^{2}\right)$. While the two designs described above in Sections 4 and 5 were developed first on physical principles and subsequently here modelled mathematically, this new design arose as a consequence of the mathematical models described herein. Prototypes do indeed enjoy significant distortion reduction.

To see how this new design is derived, we consider adding to the noninverting input of the comparator a function $F(t)$ such that $F(t)$ is constant over each interval $n T \leq t<(n+1) T$. At present the values taken by this function over each interval are arbitrary; we shall compute the effects of $F(t)$ on the audio output spectrum, then choose it so as to cancel out the intrinsic distortion.

With this additional design feature, the audio output of the amplifier is found to be

$$
\begin{aligned}
g_{a}(t)= & -s(t)+\frac{1}{c}[(1+k) s(t)+\theta(t)]^{\prime} \\
& -\frac{1}{48 c^{2}}\left\{\left(48+24 c T-3 c^{2} T^{2}\right) \theta^{\prime}(t)+\left(48(1+k)-c^{2} T^{2}\right) s^{\prime}(t)\right. \\
& \left.+3 c^{2} T^{2}[\theta(t)-s(t)]^{\prime} s^{2}(t)\right\}^{\prime}+O\left(\epsilon^{3}\right),
\end{aligned}
$$

where $\theta(t)$ is a slowly varying function that agrees with $F(t)$ at times $t=n T$. The last line of (6.1) represents the nonlinear distortion, and it is clear that this component can be eliminated by choosing

$$
F(n T)=s(n T)
$$


and hence $\theta(t)=s(t)$. Fortunately, no further distortion is introduced by this choice for $F(t)$, and we have

$$
g(t)=-s(t)+\frac{2+k}{c} s^{\prime}(t)-\frac{12(2+k)+6 c T-c^{2} T^{2}}{12 c^{2}} s^{\prime \prime}(t)+O\left(\epsilon^{3}\right),
$$

which is free from any nonlinear distortion. As in the other models above, it is possible to choose $k$ so that the output is, to the order calculated, a delayed version of the input signal, and suffers no further distortion beyond the slight delay.

7. Nonlinearity in the carrier wave. We now consider one way in which imperfect electronic components can introduce distortion into the output. Specifically, we note that it is difficult in practice to generate a high-frequency triangular carrier wave whose slopes are precisely linear. In general the wave comprises sections of exponential functions, which approximate very closely the desired piecewise-linear profile [6]. For instance, let us suppose that instead of (2.1) we have for the carrier wave

$$
v(t)= \begin{cases}1-\frac{2\left(\mathrm{e}^{t / t_{0}}-1\right)}{\mathrm{e}^{T / 2 t_{0}}-1} \equiv v_{1}(t) & \text { for } 0 \leq t<\frac{T}{2} \\ -1+\frac{2\left(\mathrm{e}^{(t-T / 2) / t_{0}}-1\right)}{\mathrm{e}^{T / 2 t_{0}}-1} \equiv v_{2}(t) & \text { for } \frac{T}{2} \leq t<T,\end{cases}
$$

and $v(t+T)=v(t)$ for all $t$. Note that the piecewise-linear profile of (2.1) is recovered as $t_{0} / T \rightarrow \infty$.

7.1. 'Classical' class-D amplifier design. Let us first consider the effects of carrier-wave nonlinearity on the 'classical' class-D amplifier design, without negative feedback. Here switching of $g(t)$ takes place whenever $v(t)+s(t)=0$, i.e., when

$$
v_{1}\left(n T+\alpha_{n}\right)+s\left(n T+\alpha_{n}\right)=0 \quad \text { or } \quad v_{2}\left(n T+\beta_{n}\right)+s\left(n T+\beta_{n}\right)=0 .
$$

These expressions are readily rearranged to give implicit equations for the switching times:

$$
\begin{aligned}
& \alpha_{n}=t_{0} \log \left\{1+\frac{1}{2}\left[1+s\left(n T+\alpha_{n}\right)\right]\left(\mathrm{e}^{T / 2 t_{0}}-1\right)\right\}, \\
& \beta_{n}=\frac{1}{2} T+t_{0} \log \left\{1+\frac{1}{2}\left[1-s\left(n T+\beta_{n}\right)\right]\left(\mathrm{e}^{T / 2 t_{0}}-1\right)\right\} .
\end{aligned}
$$

It now follows readily from (2.27) that the audio output is

$$
g_{a}(t)=\frac{2 t_{0}}{T} \log \frac{1+\frac{1}{2}[1+s(t)]\left(\mathrm{e}^{T / 2 t_{0}}-1\right)}{1+\frac{1}{2}[1-s(t)]\left(\mathrm{e}^{T / 2 t_{0}}-1\right)} .
$$

(It is straightforward from this expression to check that $g(t) \sim s(t)$ as $t_{0} / T \rightarrow \infty$, in accordance with (2.29).) Since it follows by Taylor expansion that

$$
g_{a}(t) \sim \frac{4 t_{0}}{T} \sum_{n=0}^{\infty} \frac{1}{2 n+1}\left[\frac{\left(\mathrm{e}^{T / 2 t_{0}}-1\right) s(t)}{\mathrm{e}^{T / 2 t_{0}}+1}\right]^{2 n+1},
$$

we may in principle compute the audio spectrum, for instance, due to a sinusoidal input $s(t)$.

We contrast the result here, where $g_{a} \not \equiv s$, and there is nonlinear distortion of $O\left(T / t_{0}\right)$, with that for a perfectly piecewise-linear carrier wave, where there is no distortion to the output. 
7.2. Class-D amplifier with negative feedback. We now consider the effects of carrier-wave nonlinearity on the class-D amplifier with negative feedback (as illustrated in Figure 3.1). It turns out that, provided $T / t_{0} \ll 1$, then to leading order in the nonlinearity, the output $g_{a}(t)$ as given by (3.29) is augmented by a term of the form

$$
\frac{T^{2}}{16 t_{0}}\left(s^{3}(t)-s(t)\right)^{\prime}
$$

Note that the nonlinear distortion term here (proportional to $\left.\left(s^{3}\right)^{\prime}\right)$ can be thought of as having a different phase to that inherent in the basic amplifier design (proportional to $\left.\left(s^{3}\right)^{\prime \prime}\right)$, so one cannot be used to cancel the other.

However, if we modify the design by adding to the comparator input a quantity

$$
-\frac{1}{c t_{0}} h(t)
$$

sampled at times

$$
t=n T \quad \text { and } \quad\left(n+\frac{1}{2}\right) T,
$$

in addition to the modification proposed in Section 6, then the third-harmonic distortion term due to the carrier wave nonlinearity is cancelled, and

$$
g_{a}(t)=-s(t)+c^{-1}(2+k) s^{\prime}(t)
$$

$$
+c^{-2}\left[-\frac{1}{12}\left(12(2+k)+6 c T-c^{2} T^{2}\right) s^{\prime \prime}(t)+t_{0}^{-1}(2+k) s^{\prime}(t)\right]+O\left(\epsilon^{3}\right) .
$$

With an appropriate choice for $k$, this expression is essentially just a slightly delayed version of the input signal, i.e.,

$$
g_{a}(t)=-s\left(t-t_{1}\right)+O\left(\epsilon^{3}\right) .
$$

To achieve this simplification we take

$$
k=-1+\left(1+c T-\frac{1}{6} c^{2} T^{2}\right)^{1 / 2},
$$

then the delay is

$$
t_{1}=\frac{2+k}{c}\left(1+\frac{1}{c t_{0}}\right)
$$

To the order calculated, there is no further distortion; the delay computed here is independent of the signal amplitude or frequency.

8. Conclusions. We have developed mathematical models for a variety of classD amplifier designs. While models for the 'classical' design with no negative feedback have been known for some time $[1,4,5]$, the models presented here appear to be the first to treat in detail the negative-distortion design. One model describes the use of modulation to the carrier wave symmetry in order to reduce the intrinsic distortion of the negative-feedback design; another describes the use of a sample-and-hold device to achieve essentially the same ends. The mathematical analysis has, in each case, given theoretical backing to the design idea, and quantitative statements about the parameter sets under which the designs are effective. A new means of reducing the 
intrinsic distortion has been proposed, on the basis of the mathematical models developed, which involves the use of a sample-and-hold device, but in a manner different to that in the existing design.

One of us (BHC) has tested all three designs (i.e., those described in Sections 4, 5 and 6 ) in prototype and found them all to achieve significant reduction in harmonic distortion. The two designs involving a sample-and-hold unit are found not to work as well in practice as the carrier-wave modulation system, and are more expensive to produce. The carrier-wave modulation design is the basis of a successful commercial amplifier manufactured by Halcro Pty Ltd.

It should be noted that the models developed here do not reflect a range of important practical issues, such as the noise and stability characteristics of the designs, nor their electromagnetic emissions. The models assume perfect components, an assumption that has particularly significant shortcomings in relation to sampleand-hold devices, for which the errors are relatively severe (in comparison, say, with integrators).

The models developed in this paper appear to be the first to provide an in-depth mathematical treatment of class-D amplifiers with negative feedback, and should be capable of extension to more complicated designs that reflect more accurately actual audio amplifiers.

\section{REFERENCES}

[1] H. S. Black, Modulation theory, van Nostrand, New York, 1953.

[2] P. H. Mellor, S. P. Leigh And B. M. G. Cheetham, Reduction of spectral distortion in class $D$ amplifiers by an enhanced pulse width modulation sampling process, IEE Proc.-G, 138 (1991), pp. 441-448.

[3] L. M. Milne-Thomson, The calculus of finite differences, Macmillan, London, 1933.

[4] C. Pascual, Z. Song, P. T. Krein, D. V. Sarwate, P. Midya and W. J. Roeckner, Highfidelity PWM inverter for digital audio amplification: spectral analysis, real-time DSP implementation, and results, IEEE Trans. Power Electronics, 18 (2003), pp. 473-485.

[5] Z. Song And D. V. SARwate, The frequency spectrum of pulse width modulated signals, Signal Processing, 83 (2003), pp. 2227-2258.

[6] M. T. TAn, J. S. Chang, H. C. ChuA And B. H. Gwee, An investigation into the parameters affecting total harmonic distortion in low-voltage low-power Class-D amplifiers, IEEE Trans. Circuits and Systems I, 50 (2003), pp. 1304-1315.

[7] E. T. Whittaker and G. N. Watson, A Course of Modern Analysis, Fourth ed., Cambridge University Press, Cambridge, 1927. 